\title{
Measurement of Stochastic Entropy Production
}

\author{
C. Tietz, ${ }^{1}$ S. Schuler, ${ }^{1}$ T. Speck, ${ }^{2}$ U. Seifert,${ }^{2}$ and J. Wrachtrup ${ }^{1}$ \\ ${ }^{1}$ 3. Physikalisches Institut, Universität Stuttgart, 70550 Stuttgart, Germany \\ ${ }^{2}$ II. Institut für Theoretische Physik, Universität Stuttgart, 70550 Stuttgart, Germany
}

\begin{abstract}
Using fluorescence spectroscopy we directly measure entropy production of a single two-level system realized experimentally as an optically driven defect center in diamond. We exploit a recent suggestion to define entropy on the level of a single stochastic trajectory (Seifert, Phys. Rev. Lett. 95, 040602 (2005)). Entropy production can then be split into one of the system itself and one of the surrounding medium. We demonstrate that the total entropy production obeys various exact relations for finite time trajectories.
\end{abstract}

Entropy as the central concept in statistical physics pervades many branches of science. Well-defined and uncontested only in equilibrium, its extension to timedependent non-equilibrium phenomena has been debated since the days of Boltzmann mostly in relation to an explanation of irreversibility and a foundation of the second law of thermodynamics 11. Major progress arose with the formulation of the fluctuation theorem, which quantifies in the long time limit the probability of entropy annihilating trajectories in small systems constantly driven in a steady state [2, 3, 4, 5, 6]. Entropy production in these systems is either defined as phase space contraction rate or associated with a dissipation functional, which ultimately should describe the dissipated heat.

By introducing the notion of a stochastic entropy along a single trajectory, it has become possible both to extend the validity of the fluctuation theorem to finite times and to prove an integral fluctuation theorem for the total entropy production in arbitrarily driven systems governed by stochastic dynamics [7]. In this Letter, using our previously introduced driven two-level system [8], we measure the stochastic entropy production along single trajectories and demonstrate that it obeys various exact relations for finite times.

Fluctuation theorems for entropy production should be distinguished from related theorems like the Jarzynski relation 9]. Crooks' theorem [10], and the Hatano-Sasa relation [11]. The first two allow to extract free energy differences from non-equilibrium work measurements. Experimental tests have been performed by using a torsional pendulum [12, by mechanically stretching RNA hairpins [13, 14] as well as by driving a colloidal particle in a time-dependent harmonic 15, 16 and non-harmonic potential 17. The third one yields an exact relation for transition between different steady states from which a general Clausius inequality follows that has been tested using a driven colloidal particle 18]. All these relations address a small system embedded in a surrounding heat bath of constant temperature. In contrast, our set-up works athermally and does therefore neither involve nor require any notion of dissipated heat. While our previous work has demonstrated an exact Jarzynski-like relation for the athermal analog of "dissipated work" [8], the present paper addresses the concept of stochastic entropy production directly. The crucial difference is that the derivation of the latter requires using the actual nonequilibrium probability distribution in a general master formula [7], whereas the former involves the corresponding equilibrium distribution [19]. Still, both quantities fulfill various exact relations like an integral fluctuation theorem.

Our system is a photochromic defect center in natural IIa-type diamond. Its optical properties indicate that we are dealing with a nickel-related center [20]. It can be excited by red light responding with a Stokes-shifted fluorescence. Additionally to this "bright" state the defect exhibits a non-fluorescent "dark" state. The transition rates $a$ (from dark to bright) and $b$ (from bright to dark) depend linearly on the intensity of green and red light, respectively, turning the defect center into an effective two-level system

$$
0 \text { (dark) } \underset{b}{\stackrel{a}{\rightleftharpoons}} 1 \text { (bright) }
$$

with controllable transition rates $a$ and $b$.

Single centers were addressed with a home-built confocal microscope 21] using a dye laser (CR699, DCM) running at $680 \mathrm{~nm}$ (red light intensity $\propto b$ ) superimposed to the $514 \mathrm{~nm}$ line of an Ar-Ion laser (green light intensity $\propto a$ ) as excitation sources. While the red light was kept constant throughout the experiment, the green light was modulated using a function generator controlled acoustooptical modulator (AOM). In addition to the fluorescence of the single defect, a second Avalanche Photo Diode recorded simultaneously the alternating intensity of the green light.

The system can be found in state $n$ with probability $p_{n}$, where $n$ takes either the value 0 or 1 . To drive the system out of equilibrium, we modulate the rate $a$ (from dark to bright) according to the sinusoidal protocol

$$
a(t)=a_{0}\left[1+\gamma \sin \left(2 \pi t / t_{\mathrm{m}}\right)\right]
$$

whereas the rate $b$ is held constant. The parameters are the equilibrium rates $a_{0}$ and $b$, the period $t_{\mathrm{m}}$, and the modulation depth $0 \leqslant \gamma<1$. At a time resolution of $1 \mathrm{~ms}$ the data of the two detectors were acquired simultaneously after starting the modulation protocol for the green 

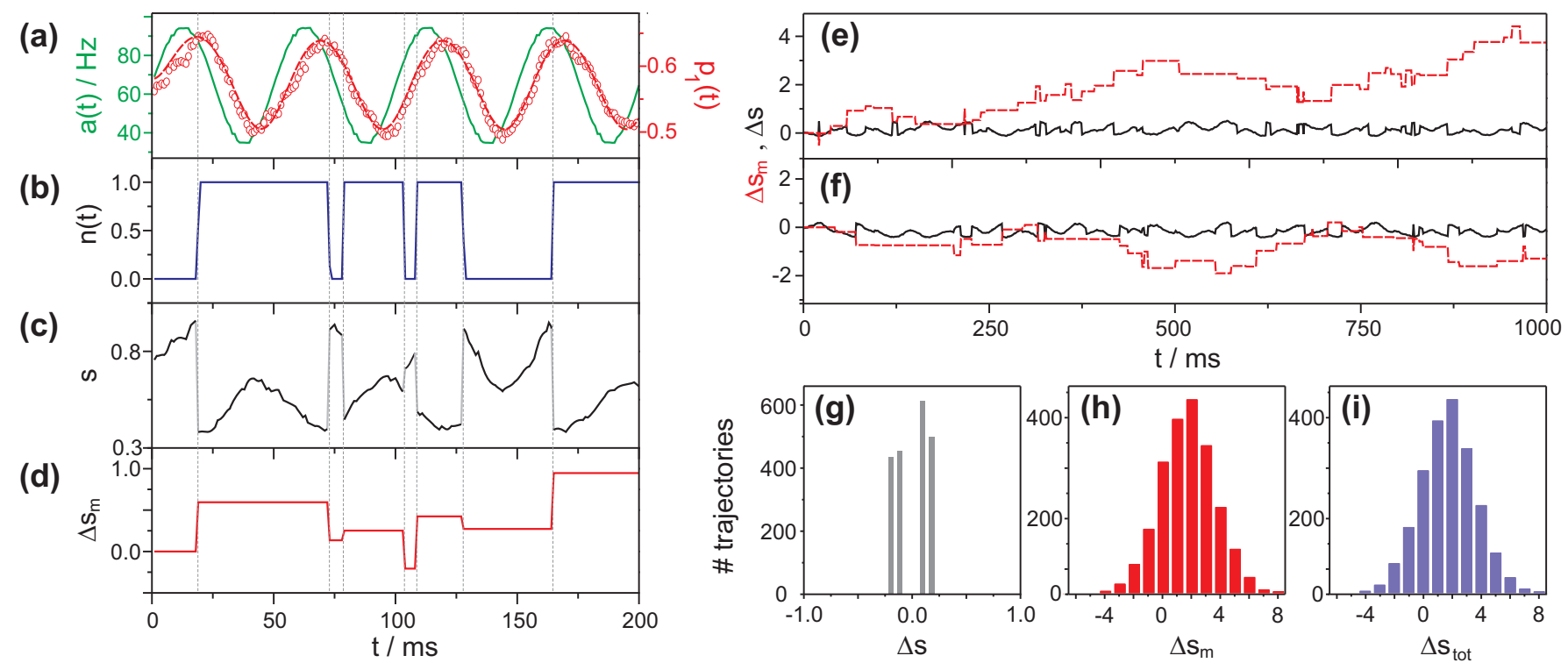

FIG. 1: Entropy production in a single two-level system with parameters $a_{0}=(15.6 \mathrm{~ms})^{-1}, b=(21.8 \mathrm{~ms})^{-1}, t_{\mathrm{m}}=50 \mathrm{~ms}$, and $\gamma=0.46$. (a) Transition rate $a(t)$ (solid/green line) and probability of the bright state $p_{1}(t)$ (red circles - measured, dashed/red line - theoretical prediction) over 4 periods. (b) Single trajectory $n(t)$. (c) Evolution of the system entropy. The gray lines correspond to jumps (vertical dotted lines) of the system whereas the dark lines show the continuous evolution due to the driving. (d) Entropy change of the medium, where only jumps contribute. (e,f) Examples of (e) entropy producing and (f) entropy annihilating trajectories. The change of system entropy $\Delta s=s(t)-s(0)$ (solid/black) fluctuates around zero without effective entropy production, whereas in (e) $\Delta s_{\mathrm{m}}$ (dashed/red) produces a net entropy over time. In (f), $\Delta s_{\mathrm{m}}$ consumes an entropy of about 1 after 20 periods. (g-i) Histograms taken from 2,000 trajectories of the system (g), medium (h), and total entropy change (i). The system entropy shows four peaks corresponding to four possibilities for the trajectory to start and end $(0 \mapsto 1,1 \mapsto 0,0 \mapsto 0$, and $1 \mapsto 1)$. The distribution $(\mathrm{h})$ of the medium entropy change has mean $\left\langle\Delta s_{\mathrm{m}}\right\rangle=1.7$ and width $\sigma_{\mathrm{m}}=3.7$, on this scale it differs only slightly from the distribution of the total entropy change (i).

laser. Following 20 periods with $t_{\mathrm{m}}=50 \mathrm{~ms}$ and a certain modulation depth $\gamma$, the system was given $1,000 \mathrm{~ms}$ of unmodulated green light to relax back into equilibrium. A sine function has been fitted to the mean intensity of the green laser to obtain the modulation depth $\gamma$. The 4 runs in this Letter contain 3 times 1,000 and once 2,000 trajectories. The modulation depths were varied from $\gamma=0.07$ to 0.46 .

A dimensionless, non-equilibrium entropy for driven systems on the level of a single stochastic trajectory has been defined in Ref. [7] as

$$
s(t)=-\ln \left[p_{n(t)}(t)\right],
$$

where the measured probability $p_{n}$ is evaluated at the actual state $n(t)$ at time $t$. Fig. 1 shows the protocol $a(t)$ together with the probability $p_{1}(t)$ to dwell in the bright state. Fig. 10 displays a sample binary trajec-

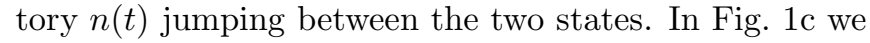
see that the evolution of $s(t)$ is governed by two effects. First, the time-dependent driving of the rates leads to an evolving probability resulting in a continuous contribution. Second, a jump between the two states gives rise to a contribution $-\ln \left[p_{+} / p_{-}\right]$, where $p_{-}$and $p_{+}$are the probabilities of the states immediately before and after the jump, respectively.
Beside the entropy of the system itself, energy exchange and dissipation lead, in general, to a change in medium entropy. For an athermal system, this change in medium entropy $\Delta s_{\mathrm{m}}$ can not be inferred from the exchanged heat. Rather it has to be defined. In Ref. [7], the choice

$$
\Delta s_{\mathrm{m}}=\ln \frac{w_{i j}}{w_{j i}}
$$

for a jump from state $i$ to state $j$ with instantaneous rate $w_{i j}$ ( $w_{j i}$ being the backward rate) has been motivated in analogy to the thermal case. In our case it becomes $\Delta s_{\mathrm{m}}=-\ln [a(t) / b]$ for a jump $1 \mapsto 0$ and $\Delta s_{\mathrm{m}}=-\ln [b / a(t)]$ for a jump $0 \mapsto 1$. As demonstrated in Fig. 1 $\mathrm{d}$, the medium entropy changes only when the system jumps, thereby balancing to some degree the change of $s(t)$.

One of the fundamental consequences of a stochastic entropy (3) is the fact that beside entropy producing trajectories also entropy annihilating trajectories exist, see Fig. 1] and f, respectively. However, in accordance with physical intuition, the latter become less likely for longer trajectories or increased system size. Entropy annihilating trajectories not only exist, they are essential to satisfy the integral fluctuation theorem [7]

$$
\left\langle\exp \left[-\Delta s_{\text {tot }}\right]\right\rangle=1 \text {. }
$$


(a)

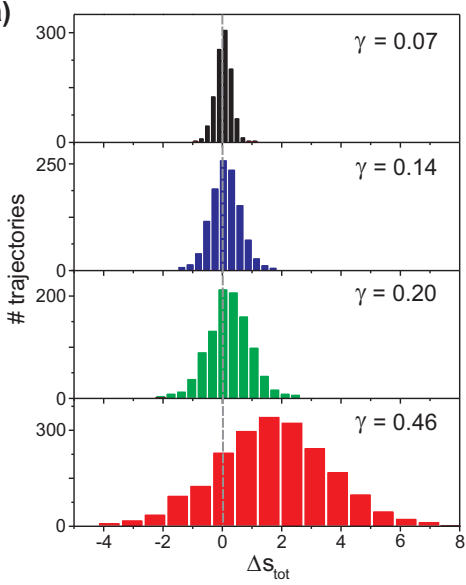

(b)

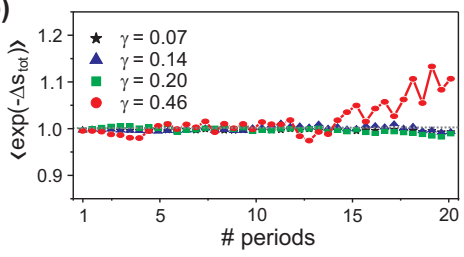

FIG. 2: Experimental test of the integral fluctuation theorem (55). (a) Probability distribution of the total entropy production of a driven two-level system. The modulation depth $\gamma$ increases from $0.07,0.14,0.20$ to 0.46 ; other parameters as in Fig. 1 The trajectory length is 20 periods with 1,000 trajectories per distribution $(2,000$ trajectories for $\gamma=0.46)$. (b) The mean $\left\langle\exp \left[-\Delta s_{\text {tot }}\right]\right\rangle$ over trajectory length for the four different modulation depths.

This theorem states that the average $\langle\cdots\rangle$ over infinitely many realizations of a process involving the total entropy change $\Delta s_{\text {tot }}=\Delta s+\Delta s_{\mathrm{m}}$ becomes unity for any trajectory length and any driving. Trajectories with $\Delta s_{\text {tot }}<0$ may occur seldom but are exponentially weighted and thus contribute substantially to the left hand side of Eq. (5). As an immediate consequence of Eq. (5) one has with $\left\langle\Delta s_{\text {tot }}\right\rangle \geqslant 0$ a consistent formulation of the second law of thermodynamic for small systems, giving $a$ posteriori support to the entropy definition (3).

Fig. 212 demonstrates how the average entropy production increases with increasing driving amplitude $\gamma$. To fulfill the constraint imposed by Eq. (5) the distributions spread, making trajectories with large negative production more likely. In Fig. 20 we present the experimental evidence for the validity of the theorem (5) analyzing 1,000 trajectories. Only for the largest modulation depth in combination with long trajectories $(t>15$ periods) a deviation from the theorem is observable. This is due to the need for larger statistics as the mean value of the entropy increases 23].

A stronger but also more special version of a fluctuation theorem is the detailed fluctuation theorem [3, 4]

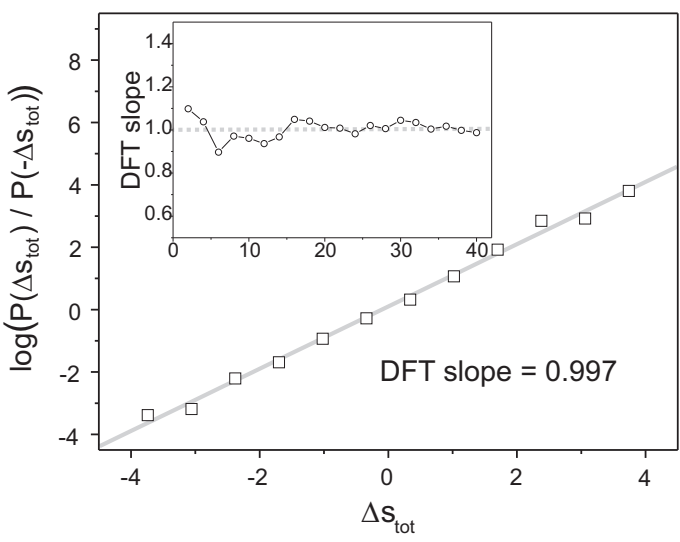

FIG. 3: Experimental test of the detailed fluctuation theorem (DFT) (6) after 20 periods with the same parameters as in Fig 1 Following Eq. (6), the slope should be 1. The inset shows the slope of the DFT as function of the trajectory length. Due to the statistics of the entropy distribution, the DFT is satisfied best for longer trajectories.

$$
\frac{P\left(+\Delta s_{\text {tot }}\right)}{P\left(-\Delta s_{\text {tot }}\right)}=\exp \left[+\Delta s_{\text {tot }}\right]
$$

Its experimental test is shown in Fig. 3. Adapted to our situation, it states that entropy annihilating trajectories with probability $P\left(-\Delta s_{\text {tot }}\right)$ become exponentially less probable compared to trajectories producing the same amount of entropy with the increasing absolute value of the entropy. This theorem was derived originally for the long-time limit in non-equilibrium steady states. However, following Crooks' reasoning 10 it even holds for periodic driving as in our experiment, provided (i) the protocol is time-symmetric, and (ii) the distribution $p_{n}(t)$ has relaxed into the corresponding periodic limit distribution. The first requirement is easily met by choosing start and end of the trajectories at $n+1 / 4$ periods. As for the second requirement, in Fig. 10 we see that the probability $p_{n}(t)$ indeed relaxes and trails with a constant phase shift behind the driving rate $a(t)$. Therefore we wait several periods before we start to record the data.

In conclusion, we have provided the first direct measurement of entropy production along single stochastic trajectories in periodically driven systems. In particular, we have shown that it is crucial to include the entropy of the system itself. While for long trajectories it remains bounded, its contribution is required for both the integral and the detailed fluctuation theorem to hold for finite times. How this stochastic entropy will contribute to a more comprehensive understanding of non-equilibrium dynamics remains to be elucidated through the future study of more complex systems.

U.S. acknowledges support in part by the NSF under Grant No. PHY99-07949 while this manuscript was finished. 
[1] J. L. Lebowitz, Physica A 263, 516 (1999).

[2] D. J. Evans, E. G. D. Cohen, and G. P. Morriss, Phys. Rev. Lett. 71, 2401 (1993).

[3] D. J. Evans and D. J. Searles, Phys. Rev. E 50, 1645 (1994).

[4] G. Gallavotti and E. G. D. Cohen, Phys. Rev. Lett. 74, 2694 (1995).

[5] J. Kurchan, J. Phys. A: Math. Gen. 31, 3719 (1998).

[6] J. L. Lebowitz and H. Spohn, J. Stat. Phys. 95, 333 (1999).

[7] U. Seifert, Phys. Rev. Lett. 95, 040602 (2005).

[8] S. Schuler, T. Speck, C. Tietz, J. Wrachtrup, and U. Seifert, Phys. Rev. Lett. 94, 180602 (2005).

[9] C. Jarzynski, Phys. Rev. Lett. 78, 2690 (1997).

[10] G. E. Crooks, Phys. Rev. E 60, 2721 (1999).

[11] T. Hatano and S. I. Sasa, Phys. Rev. Lett. 86, 3463 (2001).

[12] F. Douarche, S. Ciliberto, A. Petrosyan, and I. Rabbiosi, Europhys. Lett. 70, 593 (2005).

[13] J. Liphardt, S. Dumont, S. B. Smith, I. Tinoco Jr, and C. Bustamante, Science 296, 1832 (2002).
[14] D. Collin, F. Ritort, C. Jarzynski, S. Smith, I. Tinoco, and C. Bustamante, Nature 437, 231 (2005).

[15] G. M. Wang, E. M. Sevick, E. Mittag, D. J. Searles, and D. J. Evans, Phys. Rev. Lett. 89, 050601 (2002).

[16] D. M. Carberry, J. C. Reid, G. M. Wang, E. M. Sevick, D. J. Searles, and D. J. Evans, Phys. Rev. Lett. 92, 140601 (2004).

[17] V. Blickle, T. Speck, L. Helden, U. Seifert, and C. Bechinger, Phys. Rev. Lett. 96, 070603 (2006).

[18] E. H. Trepagnier, C. Jarzynski, F. Ritort, G. E. Crooks, C. J. Bustamante, and J. Liphardt, Proc. Natl. Acad. Sci. U.S.A. 101, 15038 (2004).

[19] U. Seifert, J. Phys. A: Math. Gen. 37, L517 (2004).

[20] A. M. Zaitsev, Optical Properties of Diamond (SpringerVerlag, Berlin Heidelberg New York, 2001).

[21] A. Gruber, A. Dräbenstedt, C. Tietz, L. Fleury, J. Wrachtrup, and C. von Borczyskowski, Science 276, 2012 (1997).

[22] D. M. Zuckerman and T. B. Woolf, Phys. Rev. Lett. 89, 180602 (2002).

[23] The discussion in Ref. 22] with respect to the change of free energy can be extended to the present case. 